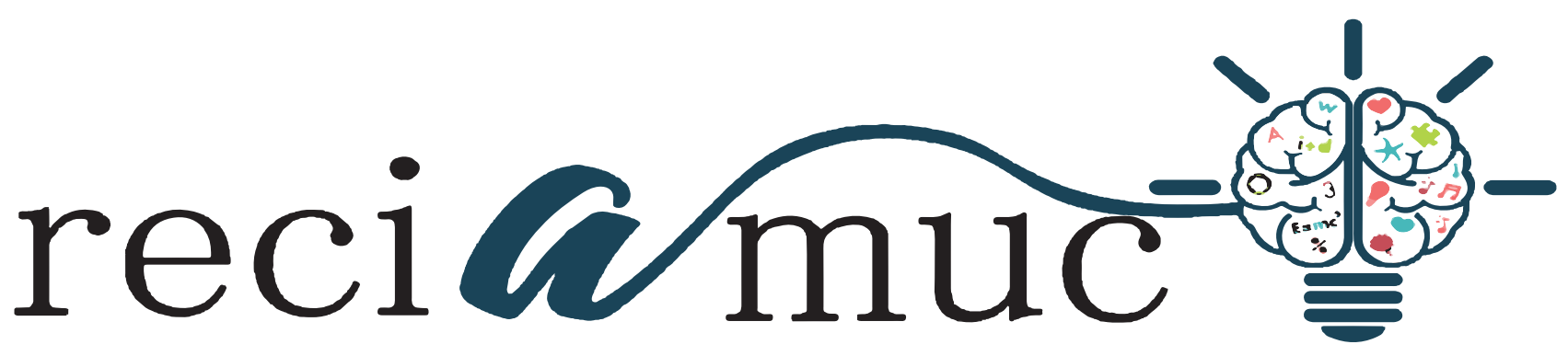

DOI: 10.26820/reciamuc/5.(4).noviembre.2021.352-357

URL: https://reciamuc.com/index.php/RECIAMUC/article/view/754

EDITORIAL: Saberes del Conocimiento

REVISTA: RECIAMUC

ISSN: 2588-0748

TIPO DE INVESTIGACIÓN: Reporte de caso

CÓDIGO UNESCO: 32 Ciencias Médicas

PAGINAS: 352-357

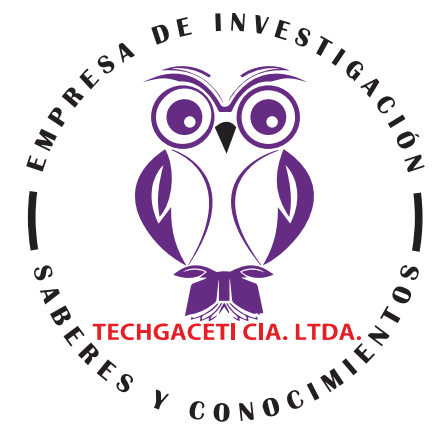

\title{
Amiotrofia Monomiélica. Apropósito de un caso
}

Monomyelic Amyotrophy. About a case

Amiotrofia monomélica. Sobre um caso

\section{Edison Andrés Orbea Jácomel; Luis Fernando Orbea Jácome²; Ana Belén Basantes Orbea3; Diego Andrés Naranjo Vásconez ${ }^{4}$}

RECIBIDO: 15/09/2021 ACEPTADO: 05/10/2021 PUBLICADO: 29/11/2021

1. Médico; Investigador Independiente; Quito, Ecuador; andres05orb@outlook.com; (D) https://orcid.org/00000001-5216-5411

2. Médico; Investigador Independiente; Quito, Ecuador; luisorbea41@gmail.com; (D) https://orcid.org/0000-00015233-7666

3. Médico; Investigador Independiente; Quito, Ecuador; anabbasant@gmail.com; (D) https://orcid.org/0000-00023998-6677

4. Médico; Investigador Independiente; Quito, Ecuador; diegoanaranjov@yahoo.es; (D) https://orcid.org/00000002-9945-666X

\section{CORRESPONDENCIA}

Edison Andrés Orbea Jácome

andres05orb@outlook.com

\section{Quito, Ecuador}

(c) RECIAMUC; Editorial Saberes del Conocimiento, 2021 


\section{RESUMEN}

La amiotrofia monomiélica es un trastorno neurológico poco común que produce atrofia de una extremidad. La afección de la extremidad superior es más frecuente que la extremidad inferior, esta entidad clínica predomina en el sexo masculino. Se presenta caso clínico de Varón, 52 años de edad, referido para evaluación por debilidad y atrofia de mano derecha. Su historia comenzó cerca de un año antes, con paresia inducida por el frio, parestesias del ante brazo seguido de debilidad en su mano que progresó hacia su antebrazo y brazo derecho. El diagnostico luego de la exploración física más los exámenes de imágenes, confirman que hay un signo de afectación de la neurona motora inferior (monomélica), y como posibilidad lejana una afectación C7-C8-T1. No se encuentran signos de afectación al plexo. En base a la literatura consultada, se desprende que la Amiotrofia Monomiélica es una enfermedad de progreso lento, que afecta a hombres después de los 30 años, aseveración que va acorde con la edad del paciente, expuesto en el caso clínico presentado en esta investigación, así como los síntomas presentados en cuanto a la disminución de fuerza en las extremidades superiores, que le puede dar un carácter de degenerativa. Tiene muy pocas opciones de tratamiento, ya que no es una enfermedad que genere dolor, sin embargo, dentro de la literatura se indica como opción terapéutica la rehabilitación física de leve a moderada, existiendo todavía muchas ambigüedad y discusión en cuanto a su efectividad en la fuerza muscular.

Palabras clave: Extremidades, Rehabilitación, Masculino, Amiotrofia, Neurológico.

\section{ABSTRACT}

Monomyelic amyotrophy is a rare neurological disorder that causes limb atrophy. The affection of the upper limb is more frequent than the lower limb, this clinical entity predominates in the male sex. A clinical case of a 52-year-old male is presented, referred for evaluation for weakness and atrophy of the right hand. His history began about a year earlier, with cold-induced paresis, forearm paresthesias followed by weakness in his hand that progressed to his forearm and right arm. The diagnosis after physical examination plus imaging tests confirm that there is a sign of lower motor neuron involvement (monomelic), and as a distant possibility a C7-C8-T1 involvement. There are no signs of plexus involvement. Based on the literature consulted, it appears that Monomyelic Amyotrophy is a disease of slow progress, which affects men after the age of 30, an assertion that is consistent with the age of the patient, exposed in the clinical case presented in this research, as well as the symptoms presented in terms of decreased strength in the upper extremities, which can give it a degenerative character. It has very few treatment options, since it is not a disease that causes pain, however, within the literature, mild to moderate physical rehabilitation is indicated as a therapeutic option, and there is still much ambiguity and discussion regarding its effectiveness in treatment. muscular strength.

Keywords: Extremities, Rehabilitation, Male, Amyotrophy, Neurological.

\section{RESUMO}

A amiotrofia monomélica é uma doença neurológica rara que causa atrofia dos membros. O acometimento do membro superior é mais frequente do que o do membro inferior, esta entidade clínica predomina no sexo masculino. É apresentado o caso clínico de um homem de 52 anos, encaminhado para avaliação por fraqueza e atrofia da mão direita. Sua história começou cerca de um ano antes, com paresia induzida pelo frio, parestesias no antebraço seguidas de fraqueza na mão que progrediu para o antebraço e braço direito. O diagnóstico após exame físico mais exames de imagem confirmam que há um sinal de envolvimento do neurônio motor inferior (monomélico), e como uma possibilidade distante de envolvimento C7-C8-T1. Não há sinais de envolvimento do plexo. Com base na literatura consultada, verifica-se que a Amiotrofia Monomélica é uma doença de evolução lenta, que acomete homens a partir dos 30 anos, afirmação condizente com a idade do paciente, exposta no caso clínico apresentado nesta pesquisa, conforme bem como os sintomas apresentados em termos de diminuição da força nas extremidades superiores, o que pode lhe conferir um caráter degenerativo. Possui pouquíssimas opções de tratamento, visto que não é uma doença causadora de dor, porém, na literatura, a reabilitação física leve a moderada é indicada como opção terapêutica, havendo ainda muita ambigüidade e discussão quanto à sua eficácia no tratamento. força muscular.

Palavras-chave: Extremidades, Reabilitação, Masculino, Amiotrofia, Neurológica. 


\section{Introducción}

Los síndromes de motoneurona inferior (MNI) son un grupo heterogéneo de enfermedades neuromusculares que se presentan clínicamente con atrofia muscular, debilidad, e hiporeflexia. Esto se deben a la disfunción y/o degeneración de la motoneurona alfa, daño que puede localizarse en la asta anterior, el axón motor y/o la mielina que lo rodea. Los síndromes de MNI pueden ser clasificados en hereditarios (ej. atrofia muscular espinal, AME), esporádicos (ej. esclerosis lateral amiotrófica, ELA) e inmunomediados (ej. neuropatía motora multifocal) (Matamala, y otros, 2021, pág. 142).

La amiotrofia monomélica benigna es un trastorno poco conocido con una presentación diferente. Epidemiológicamente, los casos reportados a nivel mundial establecen una presentación más común de miembros torácicos de forma asimétrica, predominantemente en pacientes masculinos y en países occidentales (Meza, Ojeda, \& Chávez, 2012, pág. 84). La enfermedad es más prevalente en India, Japón y otros países asiáticos, pero se han reportado muchos casos en otras partes del mundo. Un informe de la India encontró que MA comprende aproximadamente el $12,8 \%$ de las enfermedades de las neuronas motoras (Al-Ghawi, Al-Harbi, Al-Sarawi, \& Binfalah, 2016). La amiotrofia monomiélica (AMM) es un trastorno neurológico poco común que produce atrofia de una extremidad. La afección de la extremidad superior es más frecuente que la extremidad inferior por lo cual la han denominado amiotrofia monomiélica braquial (AMMB). Esta entidad clínica predomina en el sexo masculino. La AMM fue descrita inicialmente por Hirayama en 1959 quien reportó 12 pacientes jóvenes con atrofia muscular de la extremidad superior de manera unilateral (Martínez, Caro-Osorio, Gutiérrez-Jiménez, Moreno-Cuevas, \& González-Garza, 2008).
Habitualmente el diagnóstico se basa en la edad de comienzo, la presencia de antecedentes hereditarios, la distribución de las lesiones (simetría, predominio proximal o distal, afección de miembros superiores y/o inferiores) y la forma de progresión; estos datos clínicos son apoyados por los estudios de conducción nerviosa y la electromiografía. Sólo en forma reciente se han incorporado los estudios por imágenes con este objetivo (Benso, Calabrese, Jairala, \& Raimondi, 2019, pág. 73).

Los pacientes pueden presentar una disminución de los reflejos tendinosos (hipo o arreflexia) en los segmentos cervical o lumbar. Ocasionalmente, puede observarse fasciculación muscular. Hay ausencia de dolor y síntomas sensitivos. En la extremidad superior, los grupos musculares distales de las manos y del antebrazo están afectados en más del $50 \%$ de los pacientes. Los músculos de las extremidades superiores afectados con más frecuencia son los crurales. La AFB se considera una enfermedad esporádica, sin que haya otros familiares afectados por esta enfermedad al mismo tiempo. Los pacientes con AFB generalmente describen un empeoramiento de los síntomas motores en temperaturas o climas fríos (Orsini, y otros, pág. 501).

La práctica de ejercicios terapéuticos en las enfermedades de la neurona motora debe centrarse en el aumento o manejo de habilidades funcionales, sin provocar una demanda metabólica intensa en las unidades motoras restantes [3]. Hay que tener en cuenta las variables relacionadas con los ejercicios terapéuticos como la frecuencia, la duración y la intensidad. En algunos casos, pueden contribuir a la reducción de fuerza y al agravamiento de la fatiga (Orsini, y otros, 2010, pág. 317).

\section{Caso clínico}

Varón, 52 años de edad, referido para evaluación por debilidad y atrofia de mano derecha. No se describe antecedentes de infección, pero sí de exposición a produc- 
tos químicos como zinc, magnesio y cloro. Como antecedente adolece de Psoriasis por lo que ha recibido ciclos de tratamiento con antidepresivos, tranquilizantes y corticoides de uso tópico. Entre sus hábitos, tabaco veinte unidades por día, alcohol en forma ocasional, aunque en los últimos años no lo consume; antecedente familiar de enfermedades de neuromusculares es negativa.

Su historia comenzó cerca de un año antes, con paresia inducida por el frio, parestesias del ante brazo seguido de debilidad en su mano que progresó hacia su antebrazo y brazo derecho. Acudió a varios especialistas y con diagnóstico de síndrome de túnel carpiano remitido a neurocirugía.

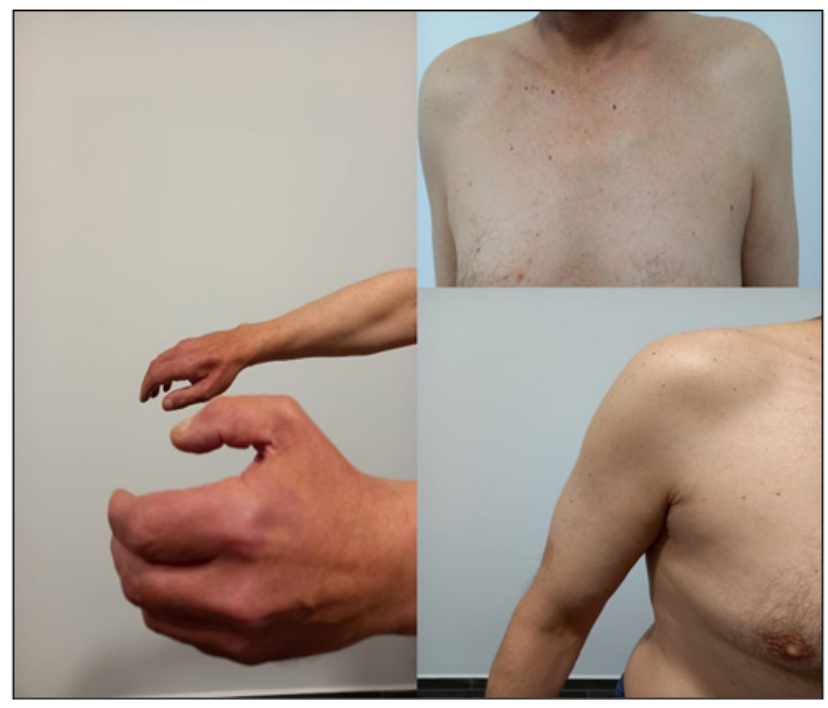

Imagen 1. Examen físico de la musculatura en el deltoides, supraescapular, trapecio, bíceps, tríceps, músculos del antebrazo y de la mano derecha

Fuente: Los Autores

Al examen se encontró hipotrofia de la musculatura en el deltoides, supraescapular, trapecio, bíceps, tríceps, músculos del antebrazo y de la mano derecha. Los reflejos miotáticos fueron normales, al igual que su sensibilidad. No hubo anormalidad en funciones cerebrales superiores, nervios craneales, sistema piramidal, sensitivo o cerebeloso.

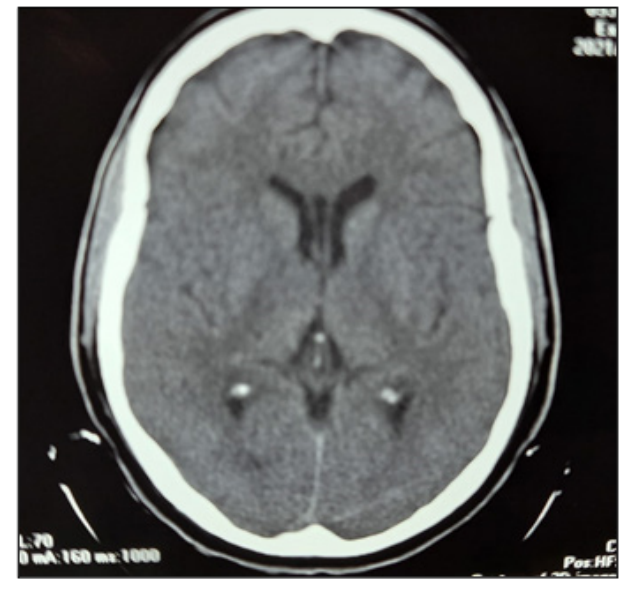

Imagen 2. TAC cerebra.

Fuente: Los Autores

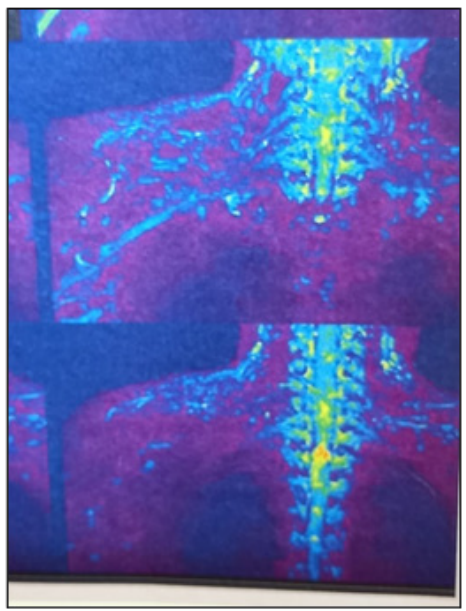

Imagen 3. Resonancia magnética de plexo braquial.

\section{Fuente: Los Autores}

La TAC cerebral y la resonancia de plexo braquial, no revelaron anormalidades, únicamente pequeños cambios en los discos intervertebrales, sin herniaciones discales. Los estudios de laboratorio de rutina que incluyó marcadores tumorales fueron normales. Anticuerpos anticardiolipina, ANCA, FR fueron negativos. La resonancia de plexo braquial dentro de parámetros normales. 


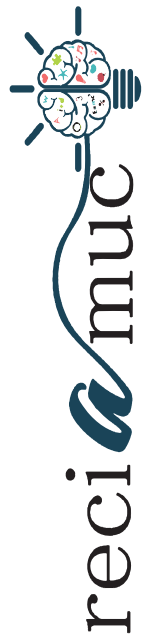

Imagen 4. Resonancia magnética de la columna cervical.

Fuente: Los Autores

El estudio electrodiagnostico de la extremidad superior derecha, presenta una disminución de la amplitud del potencial de acción musculo compuesto del cubital. La conducción motora y sensitiva de los otros nervios, es normal (mano fris 25 grados C). latencia normal de la onda $F$ del mediano y cubital.

En el deltoides, extensor común de los dedos, abductor corto del pulgar y abductor del meñique, presenta polifásicos de reinervacion, reclutamiento disminuido, con un patrón interferencial del 25 al 75\%.

\section{Discusión}

El diagnostico luego de la exploración física más los exámenes de imágenes, confirman que hay un signo de afectación de la neurona motora inferior (monomélica), y como posibilidad lejana una afectación C7-C8-T1. No se encuentran signos de afectación al plexo. En cuanto al tratamiento, en la bibliografía consultada hay opiniones encontradas en cuanto al tratamiento fisioterapéutico, ya que no se aconsejan ejercicios de alta resistencia, ya que estos pueden agravar la condición del paciente, por lo que aconsejan realizarlos de leve a moderados.

Otras terapias que han sido utilizada, están las estimulaciones eléctricas neuromuscu- lares, sin embargo, estudios de Ávila et al, han concluido que no han mejorado la fuerza muscular, sin embargo, tanto las terapias de simulación eléctrica, como rehabilitación física, al no haber tantos estudios de casos realizados, por ser una enfermedad benigna, no hay un panorama claro en cuanto a su aplicación.

\section{Conclusión}

En base a la literatura consultada, se desprende que la Amiotrofia Monomiélica es una enfermedad de progreso lento, que afecta a hombres después de los 30 años, aseveración que va acorde con la edad del paciente, expuesto en el caso clínico presentado en esta investigación, así como los síntomas presentados en cuanto a la disminución de fuerza en las extremidades superiores, que le puede dar un carácter de degenerativa. Tiene muy pocas opciones de tratamiento, ya que no es una enfermedad que genere dolor, sin embargo, dentro de la literatura se indica como opción terapéutica la rehabilitación física de leve a moderada, existiendo todavía muchas ambigüedad y discusión en cuanto a su efectividad en la fuerza muscular.

\section{Bibliografía}

Al-Ghawi, E., Al-Harbi, T., Al-Sarawi, A., \& Binfalah, M. (2016). Monomelic amyotrophy with proximal upper limb involvement: a case report. Journal of Medical Case Reports, 10(54). doi:10.1186/ s13256-016-0843-5

Benso, R., Calabrese, E., Jairala, J., \& Raimondi, E. (2019). Enfermedad de motoneurona inferior asociada al signo ojos de serpiente: Una nueva entidad clínico-radiológica. Revista Médica de Rosario, 85(2), 072-076.

Martínez, H. R., Caro-Osorio, E., Gutiérrez-Jiménez, E., Moreno-Cuevas, J., \& González-Garza, M. (2008). Amiotrofia monomiélica. Revista Mexicana de Neurociencia, 9(1), 70-73.

Matamala, J. M., Cea, G., Salinas, R., Vidal, A., López, I., \& Marileo, R. (2021). Atrofia monomiélica distal de extremidad superior. Caso clínico. Revista médica de Chile, 419(1), 142-146. doi:http://dx. doi.org/10.4067/S0034-98872021000100142 
Meza, C. G., Ojeda, G., \& Chávez, C. (2012). Amiotrofia monomélica benigna. Reporte de caso. Revista Mexicana de Medicina Física y Rehabilitación, 24(3), 84-88.

Orsini, M., De Freitas, M., Oliveira, A., Silva, J., Leite , M., \& Nascimento, O. (2010). Efectos de un programa de facilitación neuromuscular propioceptiva sobre la amiotrofia focal benigna. Rev Neurol, $51,317-8$
Orsini, M., de Freitas, M., Nascimento , O., Chieia, M., Antonioli , R., Quintanilha, G., . . Catharino, A. (s.f.). Espectro de la presentacion clinica de la amiotrofia focal benigna. Rev Neurol :501-3, 48(9), 501-3.

\section{CitAR ESTE ARTICULO:}

Orbea Jácome, E. A., Orbea Jácome, L. F., Basantes Orbea, A. B., \& Naranjo Vásconez, D. A. (2021). Amiotrofia Monomiélica. Apropósito de un caso. RECIAMUC, 5(4), 352-357. https://doi.org/10.26820/reciamuc/5.(4).noviembre.2021.352-357

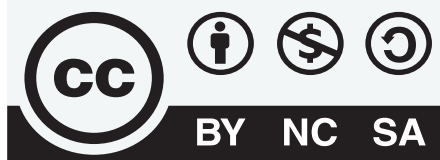

CREATIVE COMMONS RECONOCIMIENTO-NOCOMERCIAL-COMPARTIRIGUAL 4.0. 